\title{
EXOESQUELETO DE BAJO COSTE CONTROLADO POR SEÑALES EMG Y EEG ORIENTADO A TERAPIAS DE REHABILITACIÓN DE MANO
}

\author{
Óscar Fernández-Ballesteros, Javier Belmar, José M. Azorín, Eduardo Iáñez \\ Brain-Machine Interface Systems Lab, Universidad Miguel Hernández de Elche \\ Avda. de la Universidad s/n. Ed. Innova, 03202 Elche (Alicante), España \\ oscar.fernandez05@goumh.umh.es, javier.belmar@goumh.umh.es, jm.azorin@umh.es, eianez@umh.es
}

\begin{abstract}
Resumen
El objetivo principal del presente proyecto es mejorar el diseño y la estrategia de control de un exoesqueleto de mano para una terapia de rehabilitación. El exoesqueleto, impreso en $3 D$, apoya al movimiento del usuario cuando éste no esté realizando correctamente la tarea o no pueda seguir el patrón solicitado. Se ha desarrollado una estrategia de control basada en señales electromiográficas $(E M G)$ y la posición de los servomotores. Además, se ha realizado una interfaz gráfica que sirve como realimentación visual al usuario, y permite conocer cómo ha realizado la terapia. Se han realizado pruebas en tres usuarios, validando de esta forma la estrategia de control basada en EMG para el control del exoesqueleto. Finalmente, se ha implementado una interfaz cerebrocomputador para el control del exoesqueleto. Un usuario ha validado el sistema realizando imaginación motora de abrir y cerrar la mano alcanzando un $68 \%$ de acierto en la realización de la tarea en tiempo real mientras el exoesqueleto apoyaba al movimiento.
\end{abstract}

Palabras clave: exoesqueleto, EMG, BMI, estrategia de control

\section{$1 \quad$ Introducción}

Actualmente, debido a unos malos hábitos de vida, el crecimiento de la esperanza de vida y las enfermedades cerebrovasculares están en aumento. Estas pueden provocar una discapacidad motora, afectando a la movilidad de una persona. Según un artículo de ElPaís más de 300.000 personas sufren una enfermedad de este tipo en España, de las cuales en torno al $60 \%$ no pueden realizar actividades cotidianas y un $45 \%$ tienen discapacidad total o grave [1]. A su vez, el crecimiento de la esperanza de vida provoca una población más mayor, se estima que en 2050 el $35 \%$ de la población esté por encima de los 65 años [2]. Por tanto, la población será más dependiente, es por ello por lo que, los términos envejecimiento, enfermedades cerebrovasculares y dependencia están cada vez más relacionados. Estas personas ven limitadas sus capacidades para desarrollar su actividad cotidiana y requieren de una ayuda para poder realizarla.

La utilización de exoesqueletos en rehabilitación puede ser muy útil ya que permiten ser muy selectivos con el tipo de tratamiento debido a su preciso control, así como conocer en todo momento el rendimiento y los biomarcadores del movimiento, pudiendo adaptar los niveles del ejercicio de tratamiento en función de las necesidades del usuario [3]. Debido a las numerosas lesiones que se pueden producir o los diferentes diseños mecánicos o el desarrollo del sistema de control [4], numerosos exoesqueletos diferentes se pueden englobar en categorías según: la parte del cuerpo sobre la que actúa, cómo se alimentan, la movilidad de estos, los materiales usados en su fabricación y la interfaz que es usada para su manejo [5].

Actualmente, es posible establecer un canal de comunicación con el usuario usando el pensamiento, para ello, se suelen emplear técnicas no invasivas con el fin de obtener señales electroencefalográficas. Aunque actualmente las interfaces basadas en EEG no son capaces de decodificar cualquier pensamiento, si son capaces de deducir la intención del usuario en base a procesos ya conocidos [6].

En este proyecto se ha conseguido mejorar el exoesqueleto inicial, aportarle mayor ergonomía y desarrollar dos interfaces de control: una interfaz basada en señales electromiográficas (EMG) y otra cerebro-máquina (BMI). Todo ello se ha realizado con un bajo coste. Con este proyecto se aporta una posible solución a la terapia de rehabilitación de una persona.

\section{$2 \quad$ Materiales y métodos}

\subsection{Descripción del exoesqueleto}

El exoesqueleto del cual se parte es un exoesqueleto de bajo coste y activo para rehabilitación, mecánicamente simple y adaptable a diferentes 
tamaños de manos y dedos [7]. Usa tres servomotores, dos que controlan una pareja de dedos y otro el pulgar. Estos servomotores están conectados a la punta de los dedos mediante un hilo de Nylon, simulando los tendones que ayudan a realizar los movimientos de abrir y cerrar la mano. El exoesqueleto tiene una limitación del movimiento y tan sólo puede cerrar la mano hasta la mitad, en un pequeño intervalo no superior a $90^{\circ}$, como también se indica en [7]. Esta limitación se debe en parte a la flexibilidad del cable y la lejanía de los servomotores. Los modelos de los servomotores son el 3001 HB de Futaba, Hs-81 y Hs300, ambos de Hitec. Los dos primeros son analógicos y el último digital. El primero de ellos mueve los dedos índice y corazón y el digital mueve los dedos anular y meñique, mientras que el Hs-81 mueve el pulgar. Estos servomotores son ligeros y pueden mover la mano y además disponen de la rapidez necesaria ya que van a cambiar su posición cada 0.3 segundos. El exoesqueleto se puede ver en la figura 1.

\subsection{Mejoras sobre el diseño anterior}

Anteriormente los servomotores se alimentaban con 4 pilas recargables, ahora se ha utilizado la Power Bank P51 de SiliconPower. Este modelo cumple con los requisitos buscados: tamaño reducido, ligereza y que sea capaz de cubrir los picos de consumo de los servomotores.

Se ha diseñado una pieza que haga de soporte para colocar la batería. Dicha pieza, así como la batería pueden observarse en la figura 2 (C, D). Se ha aprovechado el potenciómetro interno de los servomotores para conocer la posición de estos. Para ello, se ha leído el valor de tensión en diferentes ángulos y se ha caracterizado la relación lineal entre voltaje y la posición del servomotor con un error de aproximadamente $3^{\circ}$. Esta relación se puede observar en la ecuación 1 , donde 1.87 es la relación lineal voltaje-posición. Esta posición es clave en la estrategia de control ya que permitirá conocer la posición de la mano del usuario.

$$
\text { ángulo }(\stackrel{\mathrm{o}}{)})=\operatorname{Vin} \frac{180}{1.87}(1)
$$

Se ha utilizado la medida de la posición del servomotor $3001 \mathrm{HB}$ de Futaba ya que es el más preciso en la medida y, teniendo en cuenta que la posición de la mano será la misma durante todo el movimiento, también lo será para los servomotores.

También se han diseñado unas piezas para mejorar la comodidad del exoesqueleto, su ergonomía y su facilitación a la hora de colocarse en el brazo. En esta pieza el velcro pasa por el medio y al girar se abrocha con la otra cara del velcro, pudiendo de esta forma un usuario ajustarse el velcro el mismo a su brazo. En total se han impreso 3 piezas iguales que se han colocado a lo largo del exoesqueleto donde se encontraban las fijaciones del velcro. En la figura 2 se pueden ver las piezas descritas (A) y su colocación en el exoesqueleto (B).

\subsection{Adquisición y procesamiento de la señal EMG}

Para la adquisición de la señal EMG se ha utilizado un método no invasivo mediante electrodos superficiales secos planares. Tan sólo se utiliza un canal diferencial midiendo el grupo muscular flexor del brazo derecho, separando los electrodos un par de centímetros y utilizando el codo como referencia. En la figura 3 se puede observar la localización de los electrodos, la cual no dificulta la colocación del exoesqueleto.

Para la adquisición y la etapa de acondicionamiento se ha utilizado el equipo comercial g.USBamp de g.Tec. Concretamente se ha utilizado para registrar la señal a

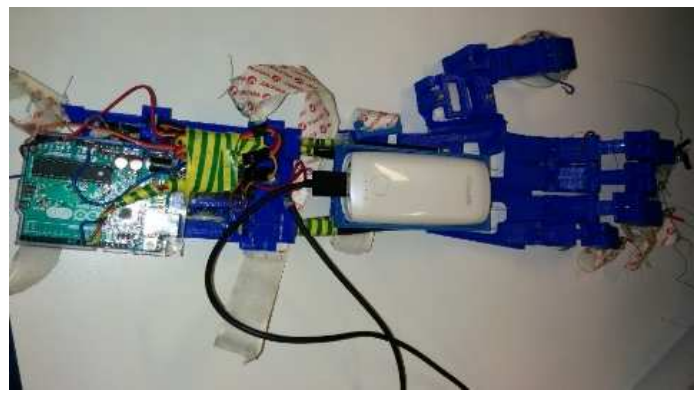

Figura 1: Exoesqueleto

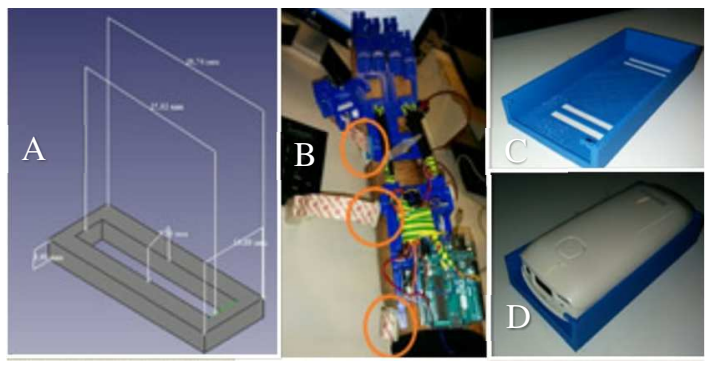

Figura 2: Piezas 3D. La imagen A es la pieza diseñada para facilitar la colocación del exoesqueleto en el brazo y la B es el exoesqueleto puesto en el brazo con las piezas descritas marcadas en naranja. La imagen $\mathrm{C}$ es la pieza para la sujeción de la batería y la D es la misma pieza con la batería encima.

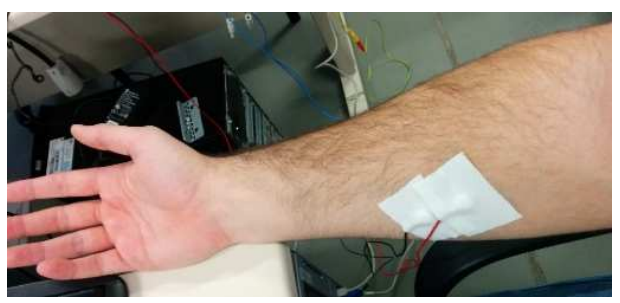

Figura 3: Localización de los electrodos 
$1200 \mathrm{~Hz}$, realizar una amplificación, digitalización y un primer acondicionamiento de la señal mediante filtrado. Para ello, el equipo primero aplica un filtrado Notch a $50 \mathrm{~Hz}$ para eliminar las interferencias de la red eléctrica. Posteriormente se aplica un filtro paso banda ya que tan sólo se va a utilizar la señal EMG en el rango frecuencial entre $5 \mathrm{~Hz}$ y $100 \mathrm{~Hz}$. Dichas frecuencias son las frecuencias de corte inferior y superior del paso banda. Con este rango frecuencial se ha comprobado que se puede conocer si el usuario realiza o no fuerza para abrir o cerrar la mano. Posteriormente la señal se amplifica y se digitaliza para enviarla al ordenador, donde se le va a aplicar un procesamiento digital a la señal.

\subsubsection{Preprocesamiento de la señal EMG}

Con el fin de eliminar la información introducida por artefactos a baja frecuencia se realiza un filtrado paso alto de fase 0 de Butterworth de orden 5 y con frecuencia de corte a $20 \mathrm{~Hz}$, posteriormente se ha aplicado una rectificación tomando el valor absoluto de la señal, y finalmente se ha realizado un filtrado paso bajo de Butterworth de fase 0 y orden 5 y frecuencia de corte $1 \mathrm{~Hz}$ con el fin de suavizar la señal y obtener la envolvente. En la figura 4 se puede observar una señal EMG adquirida y procesada como se ha comentado, en la cual se pueden apreciar 6 picos de amplitud, los cuales se corresponden a seis movimientos realizados por el usuario, siendo los centrales movimientos realizados con mayor intensidad.

\subsubsection{Procesamiento de la señal EMG en tiempo real}

Durante las pruebas experimentales es necesario conocer si el usuario está realizando fuerza o no. Para ello, se han de evaluar las últimas muestras de señal EMG, es decir, los últimos 0.3 segundos de dicha señal. Se ha implementado una estrategia de ventana deslizante, la cual almacena los datos de los últimos 3 segundos que se tienen de señal EMG y conforme se van recibiendo nuevos datos la ventana se va desplazando ese número de muestras. A la ventana de 3 segundos se le aplica el procesamiento digital que se

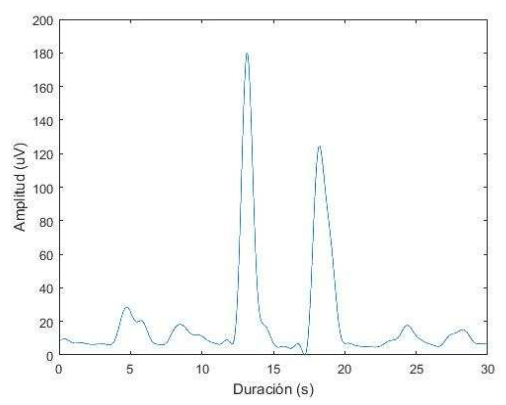

Figura 4: Señal EMG procesada ha descrito en 2.3.1 y se eligen finalmente los últimos 0.3 segundos ya que son las últimas muestras que se han medido del usuario. Posteriormente se calcula el promedio de estas últimas muestras y se comparará con el umbral, lo cual va a servir para conocer si el usuario está o no realizando fuerza.

\subsection{Caracterización del umbral de fuerza}

Para conocer si el usuario ha realizado fuerza se compara con un umbral. Para calcular dicho umbral, diferente para cada usuario, se ha diseñado una rutina en la que se compara el nivel de la fuerza con el nivel de ruido. La rutina consiste en repeticiones, dónde en cada una de ellas hay cuatro grupos de movimientos de 4 segundos cada uno. Se pide al usuario con el exoesqueleto puesto que cierre la mano de forma progresiva, después que se mantenga relajado con la mano cerrada, a continuación que abra la mano de forma progresiva, y por último que esté relajado de nuevo con la mano abierta. Durante cada movimiento se registra la señal EMG y se procesa cada una de las repeticiones por separado aplicando el procesamiento explicado en 2.3.1. Una vez realizados todos los movimientos durante las repeticiones establecidas, se calcula el máximo de cada movimiento y se hace una media del máximo de las señales de las fases de movimiento y otra media de los máximos de las fases de relax para conocer el valor medio de fuerza y de ruido, respectivamente. El objetivo es establecer un umbral que nos permita detectar cuando el usuario realiza fuerza frente a estar relajado. De forma experimental se ha obtenido que el umbral que mejores resultados ofrece es el $60 \%$ de la media de ambas fases. De esta forma, el nivel de ruido no superará el umbral, pero si lo podrá superar el usuario realizando una fuerza menor a la que ha realizado durante el proceso de caracterización.

En la figura 5 se puede observar la caracterización que se ha realizado para un usuario, en la cual se puede diferenciar la señal procesada para cada movimiento. El nivel de umbral obtenido en este caso es de 1.03 $\mu \mathrm{V}$.

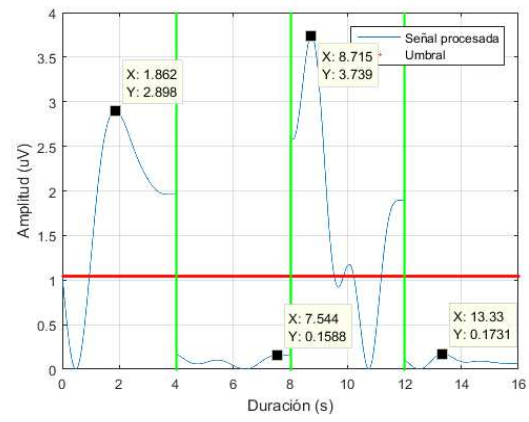

Figura 5: Caracterización del usuario 1. Las fases están separadas por una línea vertical verde. El umbra está representado como una línea roja. 


\subsection{Estrategia de control basada en EMG}

La función del exoesqueleto es la de ayudar al usuario en una terapia de habilitación, de forma que sea capaz de proporcionar la ayuda necesaria cuando este no sea capaz de seguir el ejercicio. Para ello, se ha definido una estrategia de control, empleando las señales EMG del usuario y la posición de los servomotores, lo cual ayudará a conocer en todo momento si el usuario está realizando fuerza y en qué posición tiene la mano.

Se ha definido una rutina ideal, en la que el tiempo al realizar un movimiento de abrir o cerrar la mano es de 6.9 segundos. Esta rutina simula el ejercicio que una persona sana podría hacer sin problemas.

Cada 0.3 segundos se evaluará la posición actual del usuario y si está realizando fuerza y mediante la comunicación Matlab-Arduino, se hará que el exoesqueleto sea capaz de proporcionar una respuesta correcta. La estrategia se comporta de dos posibles formas: cuando el usuario hace fuerza y cuando no.

Si el usuario está realizando fuerza el exoesqueleto sigue la posición del usuario sin proporcionar ayuda extra. Este seguimiento consiste en cambiar la posición de los servomotores $4^{\circ}$ en cada intervalo de 0.3 segundos. Se ha definido en este caso un error de posición máximo de $6^{\circ}$, de forma que, si la posición de la mano del usuario dista en más de esta cantidad de la posición ideal, el exoesqueleto proporcionará ayuda al usuario cambiando la posición de los servomotores $6^{\circ}$ hasta que el error sea nulo. De esta forma se consigue una ayuda al usuario progresiva, hasta que el error sea nulo, y un movimiento suave.

En caso de que el usuario no realice fuerza se ha definido un error máximo de posición de $15^{\circ}$, lo cual quiere decir que si el usuario no está haciendo fuerza después de un intervalo máximo de $15^{\circ}$ (1.2 segundos de inactividad), el exoesqueleto le ayudará a alcanzar la posición ideal cambiando la posición de los servomotores $6^{\circ}$ hasta llegar a la posición ideal. Esto se puede observar en la figura 6 .

Se ha realizado una simulación de la estrategia de control desarrollada, simulando seis diferentes comportamientos de un usuario. Estos resultados se pueden ver en la figura 7 .

1. El usuario realiza fuerza en todo momento

2. El usuario no realiza fuerza nunca

3. El usuario hace fuerza cerrando la mano y a mitad del movimiento deja de hacer fuerza y no vuelve a hacer fuerza durante el resto de la simulación

4. El usuario realiza fuerza cerrando la mano y a mitad de abrir deja de hacer
5. El usuario hace fuerza hasta la mitad de cerrar y durante todo el periodo en el que se abre la mano.

6. El usuario hace fuerza durante la mitad de cada movimiento

Se puede ver cómo en los diferentes casos la estrategia se comporta como se ha definido anteriormente, siendo la línea azul la posición real y la línea roja la posición ideal.

El error de posición es calculado en Matlab en cada intervalo de tiempo, y en función de estos casos envía la orden correcta a Arduino mediante un comando, el cual le indica lo que ha de hacer y contempla las posibilidades: el usuario hace fuerza y no hay error, el usuario tiene error de posición (independientemente de si hace fuerza o no).

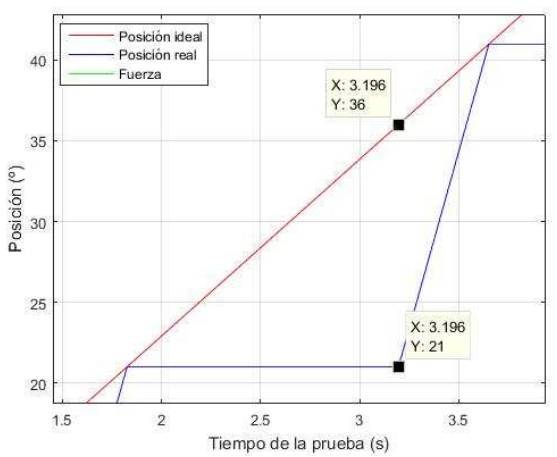

Figura 6: Error máximo y actuación si el usuario no realiza fuerza
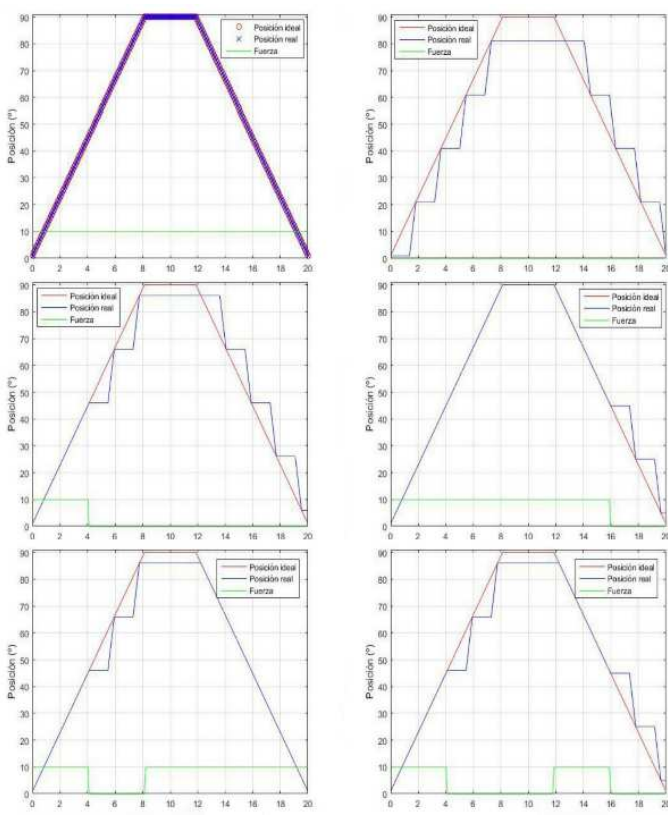

Figura 7: Resultado de la simulación de la estrategia de control 


\subsection{Protocolo experimental e interfaz gráfica}

Se ha implementado una arquitectura basada en una comunicación puerto serie entre Matlab y Arduino, en la cual hay intercambio de datos entre ambos dispositivos y Matlab y g.USBamp, en la cual Matlab tan sólo recibe datos del equipo. Matlab domina ambos dispositivos y se encarga de realizar diferentes tareas. Esta arquitectura ha sido diseñada para desarrollar la interfaz hombre-máquina implementada, la cual utiliza señales EMG y la posición de los servomotores como parámetros de entrada en la ejecución del control. La interfaz hombre-máquina implementada es un sistema en tiempo real ya que responde a los eventos de entrada en un tiempo determinado, concretamente en 0.3 segundos. Este tiempo está definido por el tiempo de adquisición del equipo g.USBamp y se ha fijado en base a un tiempo crítico de procesamiento de la señal y lectura de posición de los servomotores. Esta medida del tiempo crítico es empírica, y en base a las medidas más desfavorables (más un pequeño margen) se ha fijado la periodicidad del sistema.

Se ha desarrollado también una interfaz gráfica que permite al usuario ver el movimiento que está realizando. La interfaz desarrollada se compone de tres fases: fase de conexión, fase de operación y fase de desconexión.

\subsubsection{Fase de conexión e inicio de la interfaz}

Esta fase está definida al principio del ejercicio con el fin de realizar la conexión entre los diferentes dispositivos e iniciar la interfaz gráfica la cual consiste en dos curvas: real e ideal, ambas inician en la posición inicial (mano extendida). El aspecto de la interfaz gráfica se puede ver en la figura 8 , donde se definen dos curvas que simulan una mano vista de perfil, siendo los puntos rojos las articulaciones y el punto gordo central simula el pulgar. La posición de la

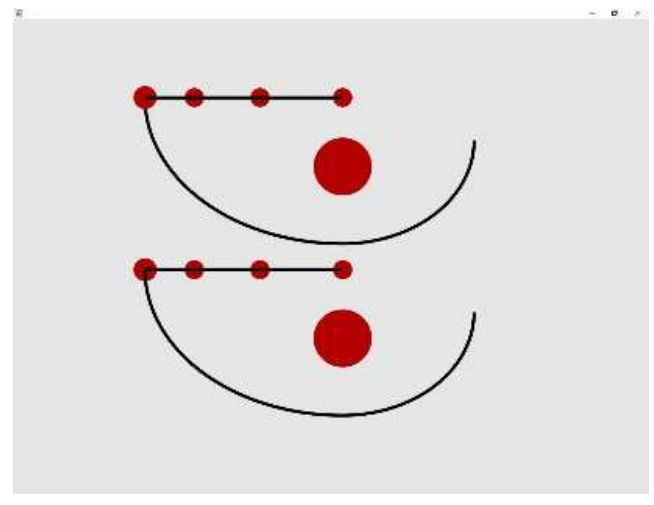

Figura 8: Interfaz gráfica desarrollada. La curva de arriba define la posición real y la de abajo la posición ideal, la cual debería seguir en todo momento el usuario. mano la marca la punta del dedo, representada con la bola roja que va siguiendo la curva.

\subsubsection{Fase de operación}

En esta fase se inicia la rutina que tiene una duración definida como el tiempo total de la prueba. El ejercicio comienza con 3 segundos iniciales en los cuales el usuario no realiza ningún movimiento, solamente se utilizan para descartar el régimen transitorio de la señal inicial de EMG. A continuación, comienza la rutina, que se va a repetir en función de la duración de la prueba. Esta rutina tiene una duración total de 28.8 segundos y se compone de: 5 segundos iniciales con la mano extendida, flexión progresiva de la mano de 6.9 segundos, 5 segundos con la mano cerrada, 6.9 segundos de extensión progresiva de la mano y otros 5 segundos con la mano extendida. Esta rutina se repetirá tantas veces como sea necesario en función de la duración total de la prueba y durante la cual, cada 0.3 segundos se lee la posición de los servomotores (posición real del usuario) y se actualizan las posiciones real e ideal en la interfaz gráfica, de forma que el usuario puede tener una realimentación visual en tiempo real gracias a esta interfaz. También se adquiere la señal EMG del usuario y se procesa mediante el procedimiento explicado en 2.3.1 con el fin de saber si realiza o no fuerza. Teniendo en cuenta la posición en la que debería de estar el usuario, en cual está y si está realizando o no fuerza, se aplica la estrategia de control explicada en 2.5.

\subsubsection{Fase de desconexión y análisis de los resultados}

Una vez finalizada la rutina se procede a desconectar los dispositivos Arduino y g.USBamp y se liberan los puertos. Además, se dibujan dos gráficas más, una de ellas mostrará el error de posición entre el usuario y la posición ideal durante la prueba; la otra mostrará el error de posición por el uso del servomotor ya que en algunos casos se leen datos del servomotor y se obtiene un cierto error entre la posición leída y la que se ha enviado. Por último, se genera una gráfica que indica la posición real e ideal del usuario en cada instante de la prueba, así como si ha hecho fuerza o no. Esta gráfica también va mostrando dicha información en tiempo real durante la fase de operación y está destinada a conocer información acerca de la actividad del usuario y a evaluar la actividad del exoesqueleto durante la realización de la terapia.

Con las gráficas obtenidas se pueden obtener conclusiones acerca de la terapia que ha estado realizando el usuario y la utilización del exoesqueleto en la misma. 


\subsection{Estrategia de control mediante BCI}

Adicionalmente, se ha implementado una interfaz cerebro-computador con la cual se ha llevado a cabo el control del exoesqueleto mediante la imaginación motora de abrir/cerrar mano del usuario. Esta interfaz está basada en la adquisición de señales EEG del usuario a $500 \mathrm{~Hz}$ mediante 31 electrodos activos no invasivos y uno de referencia en el lóbulo de la oreja. Para ello se ha utilizado el gorro de Brain Products $\mathrm{GmbH}$. La adquisición, amplificación y digitalización de estas señales se realiza mediante el equipo comercial actiChamp. Posteriormente se envían a Matlab para su procesamiento, donde se analizan dichas señales y se enviará un comando al exoesqueleto para que se mueva si el usuario está imaginando el movimiento o para que no se mueva si este no lo imagina.

La interfaz desarrollada está basada en [8] y consta de dos fases: offline y online.

\subsubsection{Fase de entrenamiento (Offline)}

Esta fase consiste en entrenar al usuario. Éste estará mirando una pantalla que le indicará qué tarea hacer: imaginar o relax; ambas tienen una duración de 10 segundos. En la primera el usuario ha de imaginar el movimiento de cerrar o abrir la mano, mientras el exoesqueleto cierra y abre la mano con el fin de ayudar a la imaginación. En la tarea de relax el usuario ha de estar relajado sin pensar nada y el exoesqueleto no se moverá. Ambas tareas se repiten hasta que cada una se ha realizado 5 veces, lo cual completa una fase de entrenamiento completa.

Posteriormente, con las pruebas de entrenamiento completadas se realiza un modelo con la ayuda de un clasificador SVM, para ello, primero se obtienen las frecuencias óptimas de los 9 electrodos que mejor detectan la zona motora del cerebro para, posteriormente, obtener el potencial espectral de cada una de estas frecuencias, calculándola en ventanas de 1 segundo.

\subsubsection{Fase online}

En esta fase se utiliza el modelo creado del usuario y se integran las mismas tareas que en la fase offline. En este caso se comparan las potencias de las frecuencias

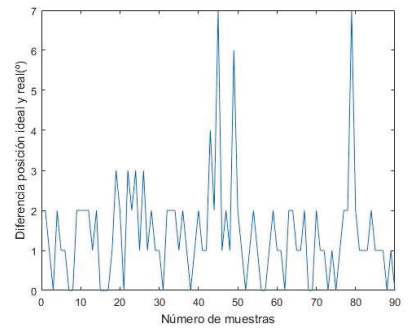

Figura 9: Error de lectura del servomotor óptimas de cada electrodo con el modelo creado y en función de si coincide o no se deducirá si el usuario está imaginando. Esta fase dispone de una interfaz gráfica que sirve tanto de realimentación visual al usuario como de información del ejercicio ya que se muestra el porcentaje de acierto y una barra que sube o baja en función de ese acierto. En esta fase cuando el usuario piense durante la tarea de imaginación y sea detectado, el exoesqueleto se moverá.

\section{Pruebas experimentales}

\subsection{Puesta en marcha del exoesqueleto}

\section{- Lecturas de posición del servomotor}

En ocasiones se manda una nueva posición al servomotor, pero en la siguiente muestra de datos se obtiene una posición que no es la deseada. Este error es conocido debido a las diferentes pruebas que se han realizado, en las cuales se han ido mandando datos y realizando su lectura $y$ se ha obtenido como conclusión que no hay error de posición en la comunicación, pero si lo hay en la lectura. Este error medio se ha estimado de forma empírica en $1.4^{\circ}$ tras haber realizado numerosas pruebas, habiendo instantes en los que el error máximo llega a $7^{\circ}$. Esto se puede visualizar en la figura 9 .

\section{- Exoesqueleto controlado con señales EMG}

En esta prueba se ha comprobado el funcionamiento de la comunicación entre los diferentes dispositivos y el movimiento del exoesqueleto cuando el usuario realiza fuerza. Para ello se ha definido una rutina donde la posición inicial es con la mano extendida y se adquiere la señal EMG, se procesa como se ha explicado en 2.3.1 y se compara con el nivel de umbral para conocer si el usuario ha realizado o no fuerza. Si el usuario ha realizado fuerza el exoesqueleto se mueve $90^{\circ}$, de forma que se cierra o se abre si el usuario realiza dicho movimiento.

\subsection{Pruebas combinando exoesqueleto y estrategia de control mediante EMG}

En esta prueba se ha utilizado la interfaz desarrollada con el sistema de control descrito en 2.5. En la figura 10 se puede ver a un usuario realizando esta prueba en la imagen A. Dicha prueba se ha realizado en tres usuarios diferentes, previamente habiendo sido caracterizado su umbral: $1.04,3.00$ y $2.10 \mu \mathrm{V}$ respectivamente. Para cada usuario se han estudiado los seis casos que se han definido anteriormente para la simulación de la estrategia de control, lo cual va a servir para comparar los resultados obtenidos en un usuario real frente a los resultados esperados idealmente. Cuando finaliza una prueba se obtienen tres gráficas definidas anteriormente en 2.5. En la 
figura 11 se pueden observar estar tres gráficas para el usuario $1 \mathrm{y}$ el caso 4 . Se puede ver en la primera gráfica, observando la línea verde, los instantes donde el usuario ha realizado fuerza y se puede observar a la perfección el sistema de control desarrollado. En la segunda se puede observar el error de posición y se puede ver cómo cuando el usuario empieza a hacer fuerza, este va un poco adelantado a la posición ideal, lo cual se resuelve manteniendo la posición de los servomotores hasta que las posiciones sean las mismas. Si se analiza la tercera gráfica de forma conjunta, se puede observar el error de lectura, el cual coincide con los máximos en los errores de posición de la gráfica anterior, es decir, los errores de lectura introducen errores de posición, los cuales han de ser solucionados por el sistema de control.

Los resultados obtenidos para las diferentes pruebas y usuarios se van a exponer en la tabla 1, en la cual se pueden ver los diferentes parámetros analizados para evaluar la estrategia de control. Para cada usuario se han realizado todas las pruebas, aunque para algunos de ellos se han obtenido resultados que no han podido ser evaluados debido a errores en las gráficas obtenidas. Estos casos han sido marcados con un guion en dicha tabla.

\subsection{Prueba con la estrategia BCI}

Se ha realizado una prueba a un usuario sano de 23 años integrando el exoesqueleto controlado con la interfaz BCI. En la figura 10 se puede observar a dicho usuario realizando la prueba con el gorro de EEG y el exoesqueleto en su brazo derecho. Se han realizado 5 sesiones de la fase offline para la creación del modelo. Para ello, se han empleado 9 electrodos, de los cuales se ha calculado su frecuencia óptima, que es en la que se ha detectado una mayor diferencia en la potencia espectral entre la señal de imaginación y la de relax. En la tabla 3 se pueden ver dichas frecuencias, y como se puede observar, son bastante estables y están en la banda beta, ya que se encuentran entre 19 y $27 \mathrm{~Hz}$. Posteriormente, se ha realizado una validación cruzada entre las 5 sesiones de entrenamiento obteniendo resultados iniciales aceptables. Después se ha utilizado el modelo del usuario en la fase online y se han realizado 3 sesiones. En la tabla 4 se observan los resultados obtenidos. Cabe destacar que el usuario ha mejorado durante la prueba, llegando a alcanzar un $76,2 \%$ de precisión durante la tarea de imaginación, y mejorando también los resultados obtenidos en offline (Tabla 4).

\section{Conclusiones}

Se ha partido de un exoesqueleto que tenía unas limitaciones iniciales: en cuanto al movimiento de la mano y la elasticidad del cable, que provoca que el usuario disponga de cierta holgura y pueda mover la mano libremente sin resistencia. Estas limitaciones se han minimizado lo máximo posible, consiguiendo sacar el máximo rendimiento al exoesqueleto. Para ello, se ha implementado un sistema de control mediante señales EMG, para que cuando el usuario

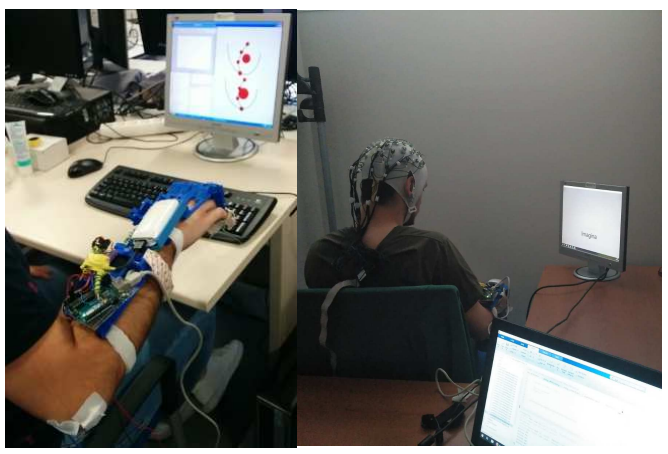

Figura 10: Usuarios realizando una prueba con la estrategia de control EMG (izquierda) y con la estrategia de control BCI (derecha)
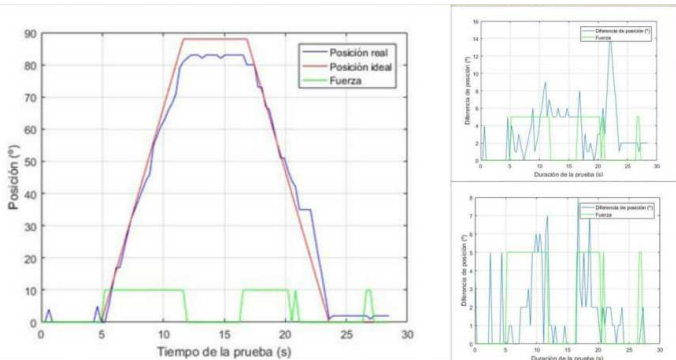

Figura 11: Resultados de la prueba con la interfaz para el usuario 1 caso 4 .

Tabla 1: Resultados de las pruebas EMG

\begin{tabular}{c|c|c|c|c|c|c}
\multirow{2}{*}{ Caso } & \multicolumn{3}{|c|}{ Error de posición $\left({ }^{\circ}\right)$} & \multicolumn{3}{c}{ Error de lectura $\left({ }^{\circ}\right)$} \\
\cline { 2 - 7 } & User:1 & User:2 & User:3 & User:1 & User:2 & User:3 \\
\hline 1 & 4,65 & - & - & 1,33 & - & - \\
\hline 2 & 6,99 & 6,50 & 7,58 & 1,16 & 1,17 & 1,09 \\
\hline 3 & 5,80 & 4,09 & 5,32 & 2,30 & 1,72 & 1,05 \\
\hline 4 & 3,20 & - & 5,68 & 1,44 & - & 1,74 \\
\hline 5 & 4,00 & 5,40 & 3,50 & 2,23 & 1,27 & 1,45 \\
\hline 6 & 5,41 & 5,09 & 6,70 & $1 ., 10$ & 1,94 & 1,30
\end{tabular}

Tabla 2: Resultados prueba offline

\begin{tabular}{c|c|c|c} 
Sesión & Imaginación & Relax & Total \\
\hline Total & $68,2 \pm 13,1$ & $55,6 \pm 11,3$ & $61,9 \pm 5,3$
\end{tabular}

Tabla 3: Frecuencias óptimas

\begin{tabular}{c|c|c|c|c|c|c|c|c|c} 
Canal & CZ & CP1 & CP2 & C1 & C2 & C3 & C4 & FC1 & FC2 \\
\hline Fr. (Hz) & 27 & 20 & 18 & 27 & 19 & 23 & 19 & 22 & 19
\end{tabular}

Tabla 4: Resultados prueba online

\begin{tabular}{c|c|c|c} 
Sesión & Imaginación & Relax & Total \\
\hline 1 & 86,2 & 48,5 & 67,3 \\
\hline 2 & 69,2 & 56,9 & 63,1 \\
\hline 3 & 73,1 & 79,2 & 76,2 \\
\hline Total & $76,2 \pm 8,9$ & $61,5 \pm 15,9$ & $68,8 \pm 6,7$
\end{tabular}


realice fuerza, el exoesqueleto lo siga, de forma que se reduzca el error de posición debido a la holgura de movimiento libre. En base a los resultados obtenidos en las pruebas, se puede afirmar que el sistema de control empleado funciona correctamente, teniendo en cuenta un cierto error en algunas medidas en la lectura de posición de los servomotores. Si el error en la lectura no es prolongado en el tiempo el sistema de control tiene una buena precisión, por debajo de los $6^{\circ}$ de error. Con ello, se ha demostrado que esta estrategia funciona dentro de unos márgenes de error debidos a estas lecturas y podría aplicarse en una terapia de rehabilitación. Esta lectura y el propio movimiento del exoesqueleto son una limitación debida a los servomotores y podría solucionarse usando otro modelo u obteniendo la posición de los servomotores aplicando otras técnicas, obteniendo así un control más preciso. Además, se ha conseguido usar un bajo coste ya que el exoesqueleto y las piezas han sido impresas en una impresora 3D, los servomotores cuestan en torno a los $10 €$ y la batería $14 €$.

Finalmente, se ha evaluado una interfaz cerebral como alternativa, o complementaria, a la interfaz hombre máquina para controlar el exoesqueleto, obteniendo un $68.8 \%$ en acierto total, comprobando la mejora del usuario conforme avanzaba la prueba. De esta forma, se aportan dos interfaces para controlar el exoesqueleto como alternativa a las terapias de rehabilitación tradicionales.

Para futuros trabajos se podría mejorar la conectividad del exoesqueleto mediante una tecnología inalámbrica, como podría ser $\mathrm{WiFi}$; también se podría mejorar el sistema de control como se ha comentado anteriormente. Por último, se podrían realizar más pruebas con ambas interfaces en usuarios finales $y$ evaluar los resultados obtenidos.

\section{English summary}

\section{LOW COST EXOSKELETON CONTROLLED BY EMG SIGNALS ORIENTED TO REHABILITATION THERAPIES OF HAND}

\author{
Abstract \\ The main objective of this project is to improve the \\ design and the control strategy of a hand exoskeleton \\ for a rehabilitation therapy. This exoskeleton, 3D \\ printed, support the movement of a user when they are \\ not performing properly the task or they cannot follow \\ the requested pattern. A control strategy based in \\ electromyographic signals (EMG) and the \\ servomotors' position has been developed. Moreover, \\ a graphic interface which serves as feedback to the \\ user has been developed, allows to know the
}

performance of the therapy. Three users validate the control strategy based on EMG signals to control the exoskeleton. Finally, a brain-computer interface to control the exoskeleton has been developed. A user validates the system by performing motor imagination of opening-closing the hand reaching a $68 \%$ of success rate performing a real time experiment while the exoskeleton supports their movements.

Keywords: exoskeleton, EMG, BMI, control strategy

\section{Referencias}

[1] Girona, Carmen, (2009). La atención del daño cerebral es insuficiente y desigual en España. Artículo en el periódico digital El País. Disponible

en: https://elpais.com/diario/2009/01/13/salud/1231 801201_850215.html

[2] Young, A. J., \& Ferris, D. P. (2017). State of the art and future directions for lower limb robotic exoskeletons. IEEE Transactions on Neural Systems and Rehabilitation Engineering, 25(2), 171-182.

[3] Destarac, Marie (2018). Desarrollo actual de exoesqueletos de rehabilitación. Disponible en: https://marieandredestarac.wordpress.com/2018 /05/22/desarrollo-actual-de-exoesqueletos-derehabilitacion/

[4] Anam, K., \& Al-Jumaily, A. A. (2012). Active exoskeleton control systems: State of the art. Procedia Engineering, 41, 988-994.

[5] Marinov, Bobby (2015). Types and classifications of exoskeletons. Disponible en: https://exoskeletonreport.com/2015/08/typesand-classifications-of-exoskeletons/

[6] Azorín (2017), JM, Interfaces cerebro-máquina basadas en señales electroencefalográficas. Lección inaugural curso académico del curso 2017-2018. Disponible en: http://protocolo.umh.es/files/2017/08/Lecci\%C3 $\%$ B3n-Inaugural.pdf

[6] Wilms, C., Rodríguez-Ugarte, M., Iánez, E., \& Azorín, J. M. (2018). Low cost 3d-printed hand exoskeleton controlled by a BCI. Actas de las XXXIX Jornadas de Automática, Badajoz, 5-7 de septiembre de 2018.

[7] Rodríguez-Ugarte, M., Iáñez, E., Ortiz, M., \& Azorin, J. M. (2018). Improving real-time lower limb motor imagery detection using tDCS and an exoskeleton. Frontiers in neuroscience, 12.

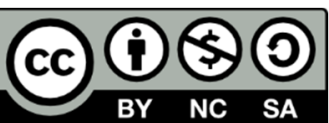
(C) 2019 by the authors. Submitted for possible open access publication under the terms and conditions of the Creative Commons Attribution CC BY-NC-SA 4.0 license (https://creativecommons.org/licenses/bync-sa/4.0/deed.es). 